\title{
Pericarditis, CTCAE
}

National Cancer Institute

\section{Source}

National Cancer Institute. Pericarditis, CT CAE. NCI Thesaurus. Code C55067.

A disorder characterized by irritation to the layers of the pericardium (the protective sac around the heart). 